\title{
Predictive Scoring for Severity of Acute Pulmonary Embolism: Does Timing Matter?
}

\author{
Abhishek Gadre, MD ${ }^{1}$ Himanshu Deshwal, MD ${ }^{2}$ Jamal Mahar, MD ${ }^{2}$ Divyajot Sadana, MD \\ Ihab Haddadin, MD ${ }^{3}$ Michael Tong, $\mathrm{MD}^{4}$ John R. Bartholomew, MD ${ }^{4}$ Gustavo A. Heresi, MD, MS ${ }^{5}$
}

\author{
${ }^{1}$ Department of Hospital Medicine, Cleveland Clinic Foundation, \\ Cleveland, Ohio \\ 2 Internal Medicine Residency Program, Cleveland Clinic, Foundation, \\ Cleveland, Ohio \\ 3 Imaging Institute, Cleveland Clinic Foundation, Cleveland, Ohio \\ ${ }^{4}$ Heart and Vascular Institute, Cleveland Clinic Foundation, \\ Cleveland, Ohio \\ ${ }^{5}$ Respiratory Institute, Cleveland Clinic Foundation, Cleveland, Ohio
}

Address for correspondence Gustavo A. Heresi, MD, MS, CTEPH Program, Respiratory Institute, Cleveland Clinic Foundation, 9500 Euclid Avenue, A90, Cleveland, OH 44195 (e-mail: heresig@ccf.org).

This is a retrospective review of the PERT registry at the Cleveland Clinic, a large tertiary care referral center. The study protocol was approved by the Cleveland Clinic Institutional Review Board (Study Number 16-547). All patients presenting to our health system with a confirmed diagnosis of acute PE for which PERT was activated between October 2014 and August 2016 were initially reviewed $(n=111)$. Sixty-six patients were excluded as the initial vital signs were not available in the 0 - to 6 -hour window. Thus, 45 patients were included for further analysis (-Fig. 1). We included PERT activations from emergency department (ED) $(n=36)$ and inpatient settings $(n=9)$. We hypothesized four ways of collecting vital signs in 0 to 6 hours of initial presentation to calculate four possible SPESI scores for every patient:

1. The first set of vital signs upon initial presentation to the ED (0 hour vitals), or upon onset of symptoms or signs leading to the diagnosis of acute PE in inpatient setting. SPESI calculated from this vital sign was called "SPESI at 0 hour."

2. Vital signs taken 2 hours after the 0 hour vitals ( 2 hour vitals). These were hypothesized to be the poststabilization vitals after initial resuscitation. SPESI calculated from this set of vital signs was called "SPESI at 2 hours."

3. Combination of vitals in the 0 - to 6 -hour window leading to the highest possible SPESI score (we refer to them as the "sickest vitals" for the purpose of discussion). The respective score was called the "highest SPESI."

4. Opposite to above, a combination of vitals in the 0 - to 6hour window leading to the lowest possible SPESI score (referred to as "healthiest vitals"). The respective score was called the "lowest SPESI." published online May 3, 2018
Issue Theme Recent Advances in Thrombosis and Hemostasis - Part III; Guest Editor: Sam Schulman, MD, PhD.
Copyright $\odot 2018$ by Thieme Medical Publishers, Inc., 333 Seventh Avenue, New York, NY 10001, USA. Tel: +1(212) 584-4662.
DOI https://doi.org/ 10.1055/s-0038-1642643. ISSN 0094-6176. 


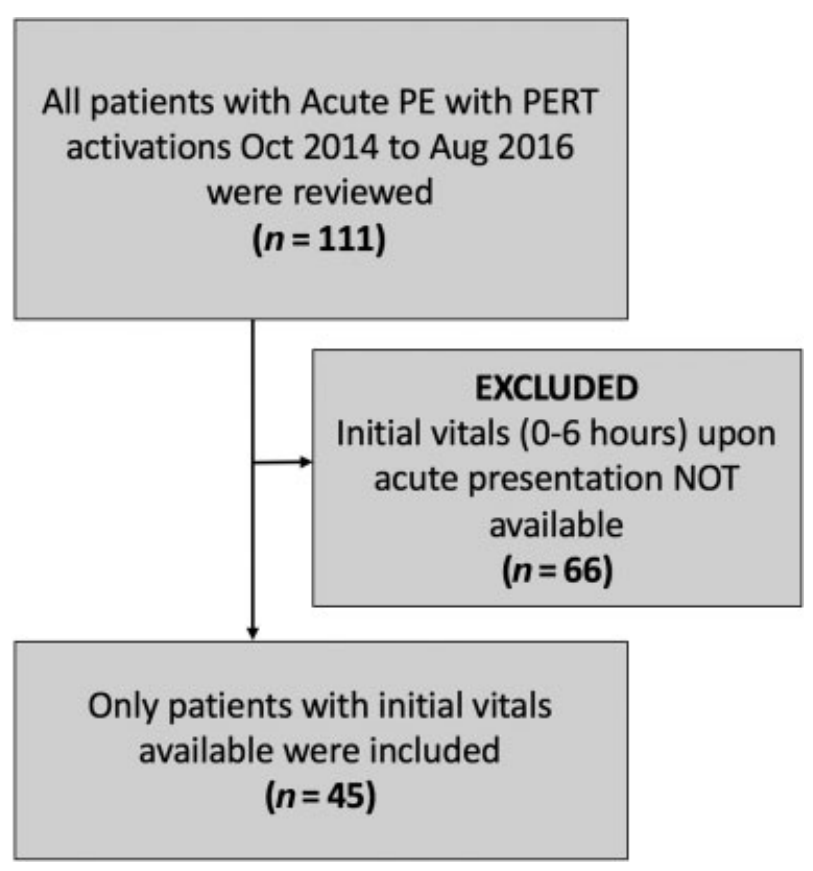

Fig. 1 Subject disposition.

The primary outcome was 30-day mortality from all causes. The mean SPESI scores at four time points were compared using Kruskal-Wallis rank test. SPESI calculated from the four hypothesized time points were used to predict the primary outcome of 30-day mortality from all causes, and $\mathrm{C}$-statistic was used to compare the area under receiver operator curve (AUROC) for the predictive models. Data analysis was performed using STATA/IC 14.1 for Mac (64bit Intel, Revision July 6, 2016).

The demographics, interventions, and outcomes are described in - Table 1. The descriptive statistics for the SPESI scores calculated at the four hypothesized time points are listed in - Table 2. The healthiest vitals led to the lowest SPESI score whereas the sickest vitals led to the highest SPESI score. There was a significant difference between the four groups of SPESI scores calculated from various time points ( $p$-value of 0.0002). Comparison of C-statistic for SPESI scores at four hypothesized time points to predict a 30-day mortality outcome is illustrated in - Fig. 2 . The "SPESI at 0 hour" was the best predictor of a 30-day mortality with an AUROC of 0.756, followed by "highest SPESI" with an AUROC of 0.753. "Lowest SPESI" is the poorest predictor of 30-day mortality with an AUROC of 0.533. The AUROC for "SPESI at 2 hours" is 0.689 . There was a statistically significant difference between AUROCs of all four groups ( $p=0.0002$ ).

To the best of our knowledge, this is the first study to address the issue of timing of vital signs and its effect of risk stratification in acute PE. This study adds to the literature that timing of vitals significantly affects the risk prediction in acute PE, and the "first vitals" (SPESI at 0 hour) or the "sickest vitals" (highest SPESI) are the best predictors of a 30-day mortality outcome. Results from the study suggests that PERT should utilize the initial vital signs to risk stratify the
Table 1 Demographic, interventions, and outcomes from the pulmonary embolism response team cohort

\begin{tabular}{|c|c|}
\hline Demographics $^{a}$ & $N=45$ \\
\hline Age, y, mean (SD) & $59.5(13.7)$ \\
\hline Female sex & $19(42.2 \%)$ \\
\hline Cancer & $13(28.9 \%)$ \\
\hline Diabetes mellitus & $13(28.9 \%)$ \\
\hline Hypertension & $20(44.4 \%)$ \\
\hline Previous pulmonary embolism & $5(11.1 \%)$ \\
\hline Chronic obstructive pulmonary disease & $7(15.6 \%)$ \\
\hline \multicolumn{2}{|l|}{ Location of pulmonary embolism } \\
\hline Saddle & $14(31.1)$ \\
\hline Main pulmonary artery & $20(44.4 \%)$ \\
\hline Lobar & $35(77.8 \%)$ \\
\hline Segmental & $32(71.1 \%)$ \\
\hline \multicolumn{2}{|l|}{ Risk level } \\
\hline Massive & $7(15.5 \%)$ \\
\hline Submassive & $28(62.2 \%)$ \\
\hline Low risk & $10(22.2 \%)$ \\
\hline \multicolumn{2}{|l|}{ Interventions } \\
\hline tPA administered & $13(28.9 \%)$ \\
\hline Catheter directed tPA & 5 \\
\hline Full dose systemic tPA & 1 \\
\hline Half dose systemic tPA & 7 \\
\hline \multicolumn{2}{|l|}{ Outcomes } \\
\hline 30-day mortality & $4(8.9 \%)$ \\
\hline Hospital mortality & $2(4.4 \%)$ \\
\hline Intracranial bleeding & 1 \\
\hline Length of stay, $d$, mean (SD) & $12.6(7.6)$ \\
\hline Length of ICU stay, $d$, mean (SD) & $5.1(6.1)$ \\
\hline
\end{tabular}

Abbreviations: ICU, intensive care unit; SD, standard deviation; tPA, tissue plasminogen activator.

${ }^{\mathrm{a}}$ Results are provided as $n(\%)$ unless otherwise stated.

patient in a more evidence-based way, and is applicable to both ED and inpatient settings. This also emphasizes the necessity of accurate documentation of the initial and the sickest vital signs.

Accurate risk stratification is crucial to determine which therapeutic approach is indicated for any given acute PE patient. ${ }^{8}$ In high-risk PE, defined as circulatory shock, there is wide agreement that early reperfusion therapy is indicated. In normotensive PE patients, risk stratification starts with the calculation of the PE severity index. Of all the risk prediction models, we chose the SPESI score for this study, because it is easy to calculate and is noninferior to the more exhaustive PESI score. During PERT calls at our institution, we struggled with the SPESI estimation in terms of timing of the vital signs for optimal predictive value. Although we have a prospective PERT registry at our institution, the specific 
Table 2 Descriptive statistics and SPESI scores from the four hypothesized time points

\begin{tabular}{|c|c|c|c|c|}
\hline \multirow[t]{2}{*}{ Timing of vitals } & \multicolumn{4}{|l|}{ SPESI score } \\
\hline & Mean (SD) & Median (IQR) & Minimum & Maximum \\
\hline $0 \mathrm{~h}$ & $1.20(1.05)$ & $1(0-2)$ & 0 & 4 \\
\hline $2 \mathrm{~h}$ & $1.22(1.16)$ & $1(0-2)$ & 0 & 5 \\
\hline Sickest vitals (0-6 h) & $1.88(1.26)$ & $2(1-3)$ & 0 & 6 \\
\hline Healthiest vitals $(0-6$ h) & $0.77(0.84)$ & $1(0-1)$ & 0 & 3 \\
\hline p-Value (Kruskal-Wallis) & 0.0002 & & & \\
\hline
\end{tabular}

Abbreviations: IQR, interquartile range; SD, standard deviation; SPESI, simplified pulmonary embolism severity index.

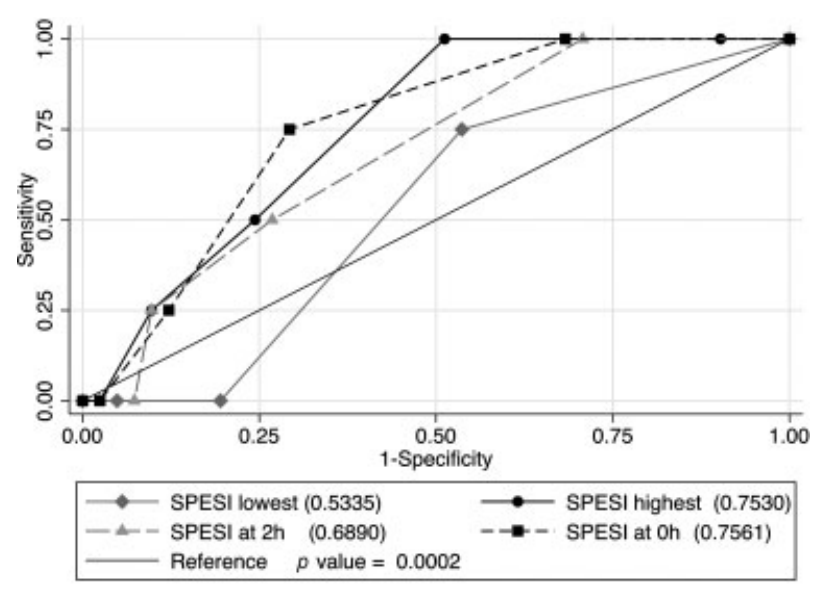

Fig. 2 Comparison of C-statistics for SPESI scores calculated from four hypothesized time points. Values in brackets represent the respective AUROCs. Abbreviations: AUROC, area under the received operator curve; SPESI, simplified pulmonary embolism severity index.

research question we had was best addressed by a retrospective review. Our hypothesis necessitated the vitals to be collected in the 0 - to 6 -hour window of acute presentation. A prospective study where a patient is enrolled after a diagnosis of acute PE is confirmed and PERT is activated, would have failed to capture vitals in the first 6 hours. Overall, our results are consistent with the original study in which the SPESI score was first described. Jiménez et al calculated a mortality rate of $8.9 \%$ for high-risk SPESI score $(\geq 1)$, and an AUROC of $0.75^{4}$ to predict mortality, which are both very similar to our findings. We recognize the limitation that this is a single-center study and 45 out of 111 patients were analyzed, and very few of those were inpatients. Being a major tertiary care referral center, our hospital receives a large number of patients from outside hospitals as transfers and direct admits. We did not have access to the initial vital signs in case of any of these patients $(n=66)$. Thus, all the aforementioned patients were excluded. The study answers a very practical clinical question and we believe the results would directly impact clinical practice.

We conclude that timing of vitals significantly affects risk stratification in acute PE. SPESI score calculated using the "first vitals" or the "sickest vitals" in the initial 6 hours are best predictors of a 30-day mortality outcome. Evidence from this study can help PERT teams to risk stratify patients in the ED.

\section{Conflict of Interest}

None.

Funding

None.

\section{Acknowledgments}

A.G. and G.H. had full access to the data and take responsibility for the integrity of the data, content of the manuscript, and accuracy of the data analysis in this original research. A.G., H.D., J.M., D.S., I.H., M.T., J.B., and G.H. contributed substantially to the study design, interpretation of results, and writing of the manuscript.

\section{References}

1 Kabrhel C, Rosovsky R, Channick R, et al. A multidisciplinary pulmonary embolism response team: initial 30-month experience with a novel approach to delivery of care to patients with submassive and massive pulmonary embolism. Chest 2016;150 (02):384-393

2 Barnes GD, Kabrhel C, Courtney DM, et al; National PERT Consortium Research Committee. Diversity in the pulmonary embolism response team model: an organizational survey of the National PERT Consortium Members. Chest 2016;150(06): 1414-1417

3 Serhal M, Haddadin IS, Heresi GA, Hornacek DA, Shishehbor MH, Bartholomew JR. Pulmonary embolism response teams. J Thromb Thrombolysis 2017;44(01):19-29

4 Jiménez D, Aujesky D, Moores L, et al; RIETE Investigators. Simplification of the pulmonary embolism severity index for prognostication in patients with acute symptomatic pulmonary embolism. Arch Intern Med 2010;170(15):1383-1389

5 Zhou XY, Ben SQ, Chen HL, Ni SS. The prognostic value of pulmonary embolism severity index in acute pulmonary embolism: a meta-analysis. Respir Res 2012;13:111

6 Aujesky D, Obrosky DS, Stone RA, et al. Derivation and validation of a prognostic model for pulmonary embolism. Am J Respir Crit Care Med 2005;172(08):1041-1046

7 Bova C, Sanchez O, Prandoni P, et al. Identification of intermediate-risk patients with acute symptomatic pulmonary embolism. Eur Respir J 2014;44(03):694-703

8 Alviar CL, Heresi GA. Thrombolysis in submassive pulmonary embolism: finding the balance. Cleve Clin J Med 2016;83(12): 933-936 